\title{
Justicia restaurativa y su relación con la empatía y los valores Sociales*
}

\section{The Relationships between Restorative Justice, Empathy and Social} Values

Recibido: 27 Diciembre 2019 | Aceptado: 29 Noviembre 2020

\author{
FRANCISCA LOZANO ESPINA \\ Universidad Nacional de Educación a Distancia, España \\ ORCID: https://orcid.org/0000-0002-7811-5567 \\ ItZiar FERnÁndez SedAnO ${ }^{a}$ \\ Universidad Nacional de Educación a Distancia, España \\ ORCID: https://orcid.org/0000-0002-6905-2111
}

a Autor de correspondencia. Correo electrónico: ifernandez@psi.uned.es

Para citar este artículo: Lozano, F., \& Fernández, I. (2021). Justicia restaurativa y su relación con la empatía y los valores Sociales. Universitas Psychologica, 20, 1-14. https://doi.org/ 10.11144/Javeriana.upsy20.jrre

\section{RESUMEN}

Con el propósito de conocer cómo se relacionan los indicadores psicosociales, caracterizados por la empatía y los valores sociales, se realizó un estudio cuasiexperimental de Justicia Restaurativa (JR) en el que participaron 187 sujetos pertenecientes a la población general, con una media de edad de $40.27(D E=12.32)$ años. Éstos fueron asignados al azar a un supuesto sobre JR que contenía 3 condiciones (desafiante, arrepentimiento e impunidad) y cuestiones sobre la gestión del delito, como son: el castigo, el diálogo, la responsabilización y la reparación. Además, los participantes cumplimentaron escalas sobre emociones negativas $(\alpha=0.81)$, empatía $(\alpha=0.72)$ y valores sociales $(\alpha=$ 0.76). A través de análisis correlacionales (. de Pearson) y de comparación de medias (prueba $t$ y ANOVA) se constató que la emoción que se siente con más intensidad, ante el delito que sufre otra persona, es el enfado, existiendo diferencias en función de las actitudes desafiante y de arrepentimiento mostradas por el agresor, para el conjunto de emociones negativas, así como para la posibilidad de aceptar el diálogo entre las partes. El hecho de poseer alta amabilidad empática hace que se faciliten los procesos de JR caracterizados por la responsabilización y la reparación, mientras que los valores de conservadurismo (tradición y seguridad) se vincularon con el castigo. Todos estos hallazgos permiten avanzar en el estudio de la JR, la empatía y los valores sociales.

Palabras clave

justicia restaurativa; delito; amabilidad empática; sufrimiento empático; valores sociales.

\section{ABSTRACT}

The aim of this study was to know the relationship between psychosocial factors (empathy and social values) and Restorative Justice (RJ). A quasiexperimental study was carried out with 187 subjects $(M=40.27$, $\mathrm{SD}=12.32$ years). Participants were randomly assigned to an assumption about RJ with 3 conditions (defiant, repentance, and impunity) and questions regarding crime management such as: punishment, dialogue, accountability and reparation. In addition, subjects completed several scales on negative emotions $(\alpha=0.81)$, empathy $(\alpha=0.72)$ and social values $(\alpha=0.76)$. Correlational analyzes (Pearson's $r$ ) and comparison of means ( $t$ test and ANOVA) were performed. The results confirmed 
that anger is the emotion most intensely felt when people are witness of the suffering of crime on another person. However, there are significant differences depending on the defiant and repentance attitudes shown by the aggressor, by the set of negative emotions, as well as by accepting the possibility of dialogue between the parties. When there is high empathic concern, it makes RJ processes characterized by accountability and reparation, while conservation values (tradition and security) were linked to punishment. All these findings reveal new knowledge in the study of RJ, empathy and social values.

Keywords

restorative justice; crime; empathic concern; personal distress; social values.

Durante las últimas décadas hemos sido testigos del nacimiento de una nueva forma de hacer Justicia, es el paradigma conocido como Justicia Restaurativa (JR), reparadora o restauradora. Este sistema gira en torno a tres ejes: víctima, victimario y pacificación social, frente a la justicia retributiva, que pone el énfasis principalmente en el castigo del infractor, generando frecuentemente en la víctima una doble victimización o victimización secundaria. El fracaso del sistema penal actual de corte retributivo es palmario. Son unánimes las voces críticas: las de la sociedad, las de los justiciables y las de las víctimas. Por lo tanto, este surgimiento se debe en parte a la necesidad de dar respuesta a una serie de carencias detectadas en el sistema de justicia tradicional, el cual no repara a las víctimas, no las escucha, no las atiende en sus verdaderas necesidades; en el caso de los infractores, nada les enseña, resultando en muchos casos la pena inútil y estéril en sus fines resocializadores; y por último, la sociedad, que siente a la justicia como una enemiga en quien no puede confiar, dado que no aporta una respuesta adecuada al delito y es, a lo sumo, un sistema de control y falsa percepción de justicia y seguridad (Bolívar \& Vanfraechem, 2015; Braithwaite, 2002; Miguel Barrio, 2019; Zehr, 2002).

Existen muchas definiciones sobre JR, ya que puede ser concebida como un enfoque alternativo para resolver conflictos y responder a la conducta delictiva, o como una tradición basada en una serie de valores complementarios al sistema tradicional penal, o como una teoría de vanguardia social de la justicia. En este sentido, la Organización de Naciones Unidas define este concepto como un proceso en el cual la víctima, el ofensor y los miembros de la comunidad afectada por la delincuencia participan en la solución de los problemas ocasionados por ésta (United Nations, 2006). Ahora bien, ninguna de estas concepciones consigue sintetizar lo que implica la JR para todas las partes afectadas (Braithwaite, 1999; Johnstone, 2013; Maglione, 2017; Marshall, 1999; Zehr, 2005).

Lo que sí podemos constatar, según Gustafson (2018), es que los pilares básicos sobre los que se sustenta la JR son la responsabilización de la persona ofensora, la reparación a la víctima y/ o personas afectadas a través del encuentro y la implicación del contexto social en la gestión de la ofensa o delito. Los procesos de JR pueden ser de muy diversa índole, los señalados por Varona (2018) son: mediación, conferencias, círculos de sentencia o de paz (sentencing/healing circles), paneles y encuentros restaurativos.

La responsabilización, como uno de los elementos clave de la JR, se produce cuando las personas reconocen que han cometido un daño que afecta a otros, asumen las consecuencias del mismo en sus diferentes niveles de impacto y buscan fórmulas de reparación de tal daño, las cuales hagan sentir mejor a las personas afectadas. Por tanto, el concepto de responsabilización incluye los procesos de reconocimiento, asunción de consecuencias y propósito de reparación.

Por su parte, la reparación es el proceso por el cual la persona que ha sufrido un daño siente que las consecuencias del mismo se ven amortiguadas o mitigadas por el efecto de alguna acción externa, especialmente si proviene de quien cometió tal daño. Esta acción genera bienestar y disminuye el impacto del daño en los diferentes niveles del mismo.

Igualmente, es considerado que las acciones que resultan especialmente significativas a este respecto están relacionadas en mayor medida con el impacto emocional de las disculpas, el reconocimiento y el compromiso de quien cometió la ofensa -reparación simbólica o 
emocional-, que con las acciones de reparación económica -reparación material- (Bolívar \& Vanfraechen, 2015; Chapman et al., 2018; Ríos et al., 2016).

La investigación actual parte de los múltiples beneficios que la participación en las prácticas restaurativas tiene para todas las partes implicadas, especialmente en comparación con los procesos retributivos tradicionales, empleados en las sociedades occidentales para la gestión del delito y el conflicto social.

Los beneficios atribuidos a la JR tienen que ver con la minimización de tiempos y costes en el ámbito procesal, lo cual puede ser visto también como efecto pernicioso, dada la tendencia de las administraciones a sobrevalorar tales ventajas y caer en el riesgo de vaciar de contenido los procedimientos restaurativos. No obstante, la concepción de las prácticas restaurativas, basadas en el diálogo y el acercamiento personal, humanizan necesariamente el sistema judicial al acercar la cultura de paz a la sociedad y superar los planteamientos adversariales, base de la justicia tradicional. Por encima de todo, se encuentra el beneficio de las personas directamente implicadas, principalmente la víctima, la cual va a contar con el espacio, negado en el proceso penal tradicional, para expresar sus emociones y la propia experiencia del delito, sentirse atendida y escuchada, y obtener respuestas que difícilmente conseguiría a través del procedimiento judicial. Funciona del mismo modo para el victimario, que acepta la posibilidad de asumir la responsabilidad por el daño causado y de buscar fórmulas comprometidas de reparación a la persona o personas dañadas por su delito, desde una base de seguridad, obteniendo además la posibilidad de que tal reparación pueda tener consecuencias penológicas en la gestión de su delito (Maglione, 2017; Olalde, 2015; Pascual, 2012).

En esta misma línea se encuentra el metaanálisis de Sherman, Strang, Mayo-Wilson et al. (2015), realizado a partir de 519 estudios que incluían a 1.880 acusados, los cuales aceptaron participar en encuentros restaurativos o conferencing, conociendo que el objetivo de esta práctica sería el encuentro con la víctima acompañada al menos por una persona de apoyo. El infractor también cuenta con alguien de apoyo y además, podría acompañarles igualmente algún miembro de la comunidad con el objeto de describir el daño causado y buscar fórmulas para su reparación. Este metaanálisis arroja los siguientes resultados: la JR realizada a través de la conferencia, en comparación con el proceso penal tradicional, consigue que los delincuentes -que participan en dicho proceso restaurativocometan menos delitos, tras un periodo de seguimiento de 2 años. Es más, la magnitud del efecto es mayor para delitos violentos, o más graves, que para delitos contra la propiedad. Este dato confirma planteamientos de otros autores que defienden el uso de prácticas restaurativas para delitos graves (Gustafson, 2018; Staiger, 2008), y contradice el planteamiento popular, según el cual las prácticas restaurativas no parecen adecuadas para determinados delitos que pudieran provocar un importante trauma en las personas que lo sufren. La práctica precisamente ha venido a evidenciar que la JR sirve para todas las víctimas y victimarios que quieren participar en este tipo de procesos, independientemente de la tipología delictiva, tal como muestran los testimonios de personas que han participado en experiencias de corte restaurativo en España sobre delitos de terrorismo (Pascual, 2013).

Sin embargo, a pesar de los citados beneficios, existen marcadas resistencias para aceptar las prácticas restaurativas como válidas en la gestión del acto delictivo. Es por ello, que el presente estudio trata de comprender los factores psicológicos que influyen en las respuestas que las personas emiten cuando entran en contacto con el delito. Igualmente intenta explorar las necesidades que estas personas presentan a la hora de gestionar tal hecho y el grado en que precisan del castigo para percibir que se ha hecho justicia, o bien puedan considerar la posibilidad del encuentro, la reparación y el perdón como mecanismos de gestión del conflicto, complementarios al sistema retributivo tradicional.

Antes de adentrarnos en los aspectos psicológicos nos detendremos en la definición del delito, que es un hecho típicamente antijurídico, 
culpable y punible. Actos delictivos son: robo con fuerza (mediante el empleo de fuerza o de pericia para acceder al lugar o al objeto; por ejemplo: robo de un coche forzando la cerradura y haciendo el puente, romper un candado para llevarse una bicicleta), robo con violencia o intimidación (amenaza con navaja, arma, tirón del bolso o atraco), hurto (sustraer al descuido sin emplear la fuerza o la intimidación), asesinato (matar a otro mediando ensañamiento, que supone el aumento deliberado e inhumano del dolor de la víctima, o alevosía asegurando el resultado o por precio o recompensa), delitos contra la salud pública (tráfico de drogas), estafa (engañar a otro para que haga una disposición patrimonial), lesiones, agresión sexual, abuso sexual y homicidio (matar a otro sin que medie alevosía o ensañamiento o precio o recompensa).

\section{Factores psicológicos y delito}

A pesar de que, en general, los españoles creen que en el origen del delito figuran las causas sociales (familia desestructurada, desempleo, marginación, exclusión social, fracaso escolar, etc.), esto no es obstáculo para que, al mismo tiempo, como ocurre en Europa, mantengan la convicción de que el cumplimento de condenas elevadas es una parte importante de la solución frente a la delincuencia (Garrido Genovés, 2010).

Además, la falsa percepción de seguridad que emana de la aplicación de respuestas altamente punitivas ante el delito, hace que socialmente sean rechazadas, o bien no tenidas en consideración, medidas basadas en prácticas restaurativas por suponerles mayor impunidad y benevolencia para los infractores, y que sin embargo, tal y como se ha acreditado mediante la experiencia práctica, a medio y largo plazo, son más fructíferas para las víctimas, victimarios y sociedad.

Los delitos, comportamientos violentos y conflictos sociales tienen importantes consecuencias no sólo para las personas directamente afectadas, sino también para los agresores y para el conjunto de la sociedad, provocando emociones negativas de miedoansiedad, tristeza-depresión, indefensión, deseos de venganza, sensación de falta de seguridad, vergüenza-culpa y frustración. Si bien, también se pueden generar sentimientos de simpatía y cariño hacia las víctimas, conductas de solidaridad, así como la participación en actividades de reparación (Hofinger et al., 2015; Páez et al., 2011).

En general, los delitos hacen que la población presente reacciones emocionales muy intensas, entre las que también podemos incluir la empatía, que es la capacidad para ponerse en el lugar del otro y/o sentir como él (Batson, 1991). Es decir, puede ser activada por la percepción afectiva de los sentimientos de los demás o por la imaginación cognitiva de lo que han vivido y experimentado, e incluso conducir a posicionamientos enrocados en relación a cómo habría que responder ante tal delito.

Ahora bien, en relación con la empatía existe un debate teórico (Lishner et al., 2011; LópezPérez, 2012) que diferencia entre la tendencia a mostrar afectos positivos hacia otras personas (en nuestro estudio amabilidad por empatía) y el sentimiento de malestar personal, o sufrimiento por empatía, que es lo que se experimenta cuando se es testigo o se evoca el sufrimiento de otros. En realidad, en este caso no se trata de preocuparse por la otra persona, ni de ponerse en su lugar, sino de la ansiedad y estrés personal generados a partir de lo acontecido. Dichas reacciones vicarias pueden coexistir tras un delito y su gestión.

Por lo tanto, las reacciones de empatía pueden estar orientadas hacia los demás de manera que las personas sientan emociones congruentes con la situación de necesidad de las víctimas, a esta reacción se le denomina amabilidad empática e incluye la simpatía y la ternura. La simpatía se vincularía con la ayuda en la situación de necesidad actual de la víctima, mientras que la ternura se relacionaría con una ayuda más prolongada dada la situación de vulnerabilidad crónica de la víctima.

Ante el sufrimiento de otras personas también pueden aparecer reacciones de empatía focalizadas en uno mismo. Es lo que se conoce 
como sufrimiento por empatía y promueve conductas de ayuda en las que el donante recibe beneficios personales por realizarlas (Lishner et al., 2011; López-Pérez et al., 2019).

Otro factor a tener en cuenta tras la vivencia de un delito es el papel que ejercen los valores sociales, ya que pueden favorecer u obstaculizar la implementación de la JR. Páez et al. (2003), así como Fernández (2012) recogen una definición ampliamente consensuada que constata que los valores moldean la conducta social, definiendo lo que se espera que sea la realidad en un contexto y momento histórico determinado, es decir, lo deseable y compartido socialmente. Así, cuando en una sociedad se da prioridad al respeto, se sabe que son intolerables otros comportamientos que no se basen en dicho valor. La importancia de los valores sociales estriba en que pueden ajustarse a lo largo del tiempo, a medida que las personas y sociedades evolucionan.

Por su parte, Schwartz y Bilsky (1990) con el propósito de analizar los valores humanos han desarrollado un modelo teórico, clasificando los valores en diez tipos motivacionales universales que se definen en los siguientes términos: (1) Autodirección: elección independiente de actos y pensamientos. (2) Estimulación: excitación, innovación y reto. (3) Hedonismo: placer y sensación grata para uno mismo. (4) Logro: éxito personal demostrando ser competente. (5) Poder: estatus social y prestigio, control y dominio sobre personas o recursos. (6) Seguridad: seguridad, armonía y estabilidad social, en las distintas relaciones y con uno mismo. (7) Conformidad: restricción a las acciones, inclinaciones e impulsos, que pudieran molestar o perjudicar a otros, así como el cumplimiento de reglas sociales o normas. (8) Tradición: respeto, compromiso y aceptación de las costumbres e ideas que la tradición cultural o la religión prescriben. (9) Benevolencia: preservación y preocupación por el bienestar de la gente con la que se está frecuentemente en contacto y (10) Universalismo: comprensión, tolerancia, apreciación y protección del bienestar de toda la gente y de la naturaleza.

Schwartz (1992) plantea que estos diez valores se agrupan sobre dos ejes. El primer eje opone el factor de apertura al cambio (que incluye los valores de autodirección y estimulación) al de conservadurismo (valores de tradición, conformidad y seguridad). El segundo eje opone el factor de promoción personal (logro y poder) al de autotrascendencia (universalismo y benevolencia). El valor de hedonismo es compartido por los objetivos básicos de promoción personal y apertura al cambio. La Figura 1 ilustra esta taxonomía. En la p. 51 del estudio realizado por Fernández (2012) se ilustra esta taxonomía.

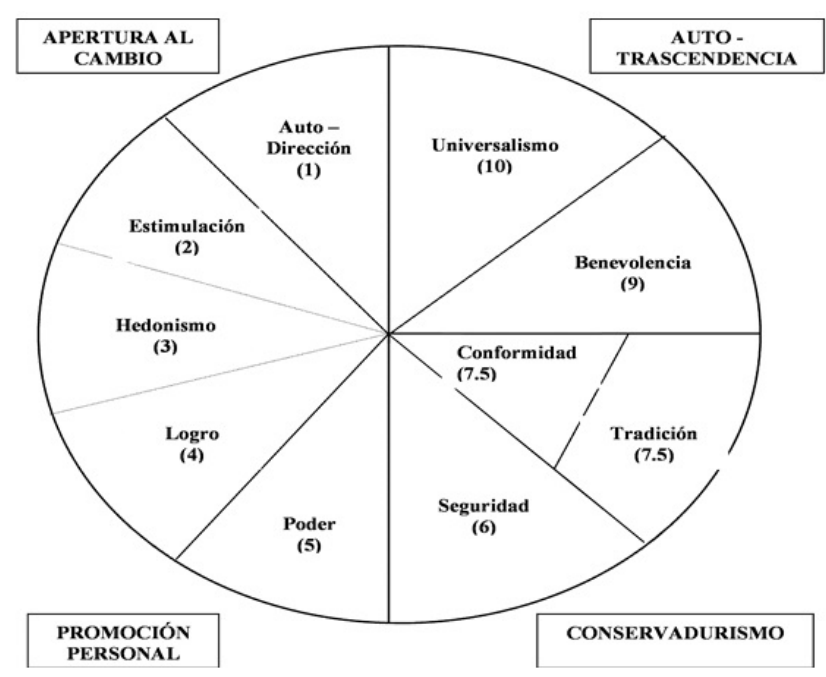

Figura 1

Valores humanos Schwartz (1992)

Además de estos valores clásicos, postulados por Schwartz, existiría una motivación social denominada quijotismo, que se define como un estado motivacional cuyo fin último es mejorar el bienestar del mundo, es decir, se debe percibir una discrepancia negativa entre el estado actual de la entidad mundo y un estado potencial (Oceja et al., 2010; Salgado, 2009).

En el presente estudio trataremos de establecer tanto la influencia de los valores sociales sobre los procesos de JR en la gestión del delito, como su relación con la amabilidad y sufrimiento empático.

Para constatar los objetivos propuestos se contará con un diseño cuasiexperimental, en el que se realizará una manipulación que consistirá 
en asignar aleatoriamente a cada participante a las condiciones desafiante, arrepentimiento e impunidad ante la gestión del delito (ver en el Apéndice A la descripción de cada una de ellas). Además, todos los sujetos contestarán a través de un autoinforme a una serie de variables psicosociales que guardan relación con las siguientes hipótesis de investigación.

H1. Los participantes que sientan un mayor número de emociones negativas, tras la resolución del delito, presentarán una menor predisposición a aceptar el diálogo, responsabilización y reparación como parte del proceso de JR, en la condición de actitud desafiante (vs. arrepentimiento e impunidad).

H2. Los participantes que consideren que el agresor debe cumplir la mayor condena posible tras el delito, tendrán una menor predisposición al diálogo y reparación como parte del proceso de JR, además mostrarán un mayor número de emociones negativas, con independencia de la condición experimental.

H3. Los participantes con alta (vs. baja) amabilidad empática tendrán mayor predisposición al diálogo, responsabilización y reparación, así como una menor preferencia por las elevadas condenas y castigos como parte del proceso de JR.

H4. Los participantes con una elevada puntuación en el valor social caracterizado por el quijotismo presentarán una mayor predisposición al diálogo, la responsabilización y reparación, así como una menor preferencia por elevadas condenas como parte del proceso de JR en la condición arrepentimiento e impunidad (vs. desafiante).

H5. Teniendo en cuenta que el sufrimiento y la amabilidad por empatía son sentimientos que hacen que se activen diferentes valores sociales, hipotetizamos que los sentimientos de amabilidad por empatía se vincularán positivamente con los valores de hedonismo, universalismo y quijotismo, mientras que el sufrimiento por empatía se relacionará con la autodirección, la tradición y la seguridad. Estas relaciones permanecerán estables para el conjunto de la muestra.

\section{Método}

\section{Participantes}

La muestra estuvo constituida por 187 sujetos, los cuales no poseían conocimientos específicos sobre JR, ni necesariamente tenían relación directa con el delito. El 36.4\% eran hombres, mientras que 119 eran mujeres (63.6\%). La media de edad fue de $40.27(D E=12.32)$, con un rango de variación comprendido entre los 19 y 74 años. Respecto a su situación laboral, la categoría con un mayor porcentaje fue la de trabajador y estudiante $(35.3 \%)$. Los participantes para cada una de las condiciones fueron asignados al azar: desafiante $(n=63)$, arrepentimiento $(n=59)$ e impunidad $(n=65)$. En la Tabla 1 se presentan las características sociodemográficas en función de cada grupo objeto de estudio.

\section{Tabla 1}

Descriptivos sociodemográficos para cada una de las condiciones experimentales y muestra total

\begin{tabular}{lrrrrr}
\hline \multicolumn{1}{c}{ Variable } & $\begin{array}{c}\text { Desafiante } \\
(\boldsymbol{n}=\mathbf{6 3})\end{array}$ & $\begin{array}{c}\text { Arrepentimiento } \\
(\boldsymbol{n}=\mathbf{5 9})\end{array}$ & $\begin{array}{c}\text { Impunidad } \\
(\boldsymbol{n}=\mathbf{6 5})\end{array}$ & $\begin{array}{c}\text { Total } \\
(\boldsymbol{n}=\mathbf{1 8 7})\end{array}$ \\
\hline Edad & $M$ & 42.38 & 40.53 & 39.08 & 40.27 \\
& $D S$ & 11.75 & 12.21 & 11.73 & 12.32 \\
Sexo & & & & \\
Hombre & $38.1 \%$ & $37.3 \%$ & $33.8 \%$ & $36.4 \%$ \\
Mujer & $61.9 \%$ & $62.7 \%$ & $66.2 \%$ & $63.6 \%$ \\
Situación laboral & & & & \\
Trabajador & $36.5 \%$ & $32.2 \%$ & $26.2 \%$ & $31.6 \%$ \\
Estudiante & $14.3 \%$ & $8.5 \%$ & $13.8 \%$ & $12.3 \%$ \\
Trabajador y estudiante & $30.2 \%$ & $40.7 \%$ & $35.4 \%$ & $35.3 \%$ \\
Pensionista & $4.8 \%$ & $5.1 \%$ & $6.2 \%$ & $5.3 \%$ \\
Pensionista y estudiante & $1.6 \%$ & - & - & $0.5 \%$ \\
Desempleado & $4.8 \%$ & $3.4 \%$ & $6.2 \%$ & $4.8 \%$ \\
Desempleado y estudiante & $7.9 \%$ & $10.2 \%$ & $12.3 \%$ & $10.2 \%$ \\
\hline
\end{tabular}

\section{Instrumentos}

Las variables y medidas aplicadas fueron las siguientes:

Justicia Restaurativa (JR). Este indicador, creado específicamente para este estudio, contiene tres supuestos en función del desarrollo del proceso de JR, a saber: (actitudes desafiantes y de arrepentimiento por parte del agresor) y una tercera condición donde se describe la situación de impunidad, ya que el proceso judicial 
no se inicia. El delito cometido consiste en robo con violencia (véase Anexo A). Además, se incluyó un ítem sobre el posible diálogo entre ofensor y ofendido ("si yo fuera la víctima de este delito desearía hablar con la persona que lo ha cometido"), otro sobre la responsabilización del agresor ("lo importante es que la persona que ha cometido el delito asuma su responsabilidad") y una última cuestión sobre la reparación a la víctima ("lo importante es que la persona que ha sufrido el delito sea reparada"). El rango de variación estuvo comprendido entre $1=$ completamente en desacuerdo y $7=$ completamente de acuerdo. Las puntuaciones medias se muestran en la Tabla 2 para cada una de las condiciones experimentales.

Castigo. Esta medida está constituida por 3 preguntas que guardan relación con el castigo que debe aplicarse al infractor tras la realización del delito (por ej. "¿impondrías la máxima condena posible?"). Su rango de variación estuvo comprendido entre 1 (completamente en desacuerdo) y 7 (completamente de acuerdo). El alfa de Cronbach fue satisfactorio para cada una de las condiciones $\left(\alpha_{\text {Desafiante }}=0.70\right.$; $\left.\alpha_{\text {Arrepentimiento }}=0.90 ; \alpha_{\text {Impunidad }}=0.78\right) . \mathrm{La}$ puntuación para cada una de las condiciones puede consultarse en la Tabla 2.

Emociones Negativas. La Escala Diferencial de Emociones de Carol Izard (DES en sus siglas en inglés, Echebarría y Páez, 1989), es una versión adaptada que contiene 10 emociones relacionadas con el tema de investigación (por ej. enfado, miedo, venganza, tristeza) siendo 5 las opciones de respuesta $(1=$ nunca, $2=$ poco, $3=$ regular, $4=$ bastante y $5=$ mucho). Esta variable se recogió tras cumplimentar la medida de JR descrita anteriormente. Se preguntó por los sentimientos del participante tras el desarrollo de la gestión del delito que ha sufrido una persona. El alfa de Cronbach fue satisfactorio para cada una de las condiciones $\left(\alpha_{\text {Desafiante }}=\right.$ $\left.0.75 ; \alpha_{\text {Arrepentimiento }}=0.86 ; \alpha_{\text {Impunidad }}=0.80\right)$, así como para el conjunto de la muestra $(\alpha=$ 0.81). Los estadísticos de tendencia central se muestran en la Tabla 2, tanto para cada una de las 10 emociones como para su conjunto.

\section{Tabla 2}

Medias (DE) de las emociones elicitadas ante la gestión por el delito, el castigo y los procesos implicados en la JR en función de la condición experimental

\begin{tabular}{lrrrrrr}
\hline \multirow{1}{*}{\multicolumn{1}{c}{ Variable }} & \multicolumn{2}{c}{$\begin{array}{c}\text { Desafiante } \\
(\boldsymbol{n}=\mathbf{6 3})\end{array}$} & \multicolumn{2}{c}{$\begin{array}{c}\text { Arrepentimiento } \\
(\boldsymbol{n}=\mathbf{5 9})\end{array}$} & \multicolumn{2}{c}{$\begin{array}{c}\text { Impunidad } \\
(\boldsymbol{n}=\mathbf{6 5})\end{array}$} \\
\cline { 2 - 8 } & \multicolumn{1}{c}{$M$} & $D E$ & \multicolumn{1}{c}{$M$} & \multicolumn{1}{c}{$D E$} & $M$ & $D E$ \\
\hline Enfado & 4.42 & 0.64 & 4.07 & 0.94 & 4.22 & 0.91 \\
Indefensión & 3.92 & 0.82 & 3.79 & 1.12 & 3.72 & 1.11 \\
Vergüenza & 2.35 & 1.33 & 2.88 & 1.53 & 2.71 & 1.34 \\
Miedo & 3.41 & 1.19 & 3.49 & 1.24 & 3.42 & 1.16 \\
Tristeza & 3.63 & 1.03 & 3.88 & 1.22 & 3.78 & 1.04 \\
Preocupación & 3.97 & 0.87 & 4.07 & 0.89 & 4.05 & 0.96 \\
Inseguridad & 3.78 & 1.02 & 3.78 & 1.2 & 3.86 & 1.17 \\
Indiferencia & 1.63 & 1 & 1.68 & 1.22 & 1.64 & 0.91 \\
Venganza & 2 & 1.13 & 2.36 & 1.28 & 2.63 & 1.32 \\
Incomodidad & 3.15 & 1.32 & 3.29 & 1.37 & 3.29 & 1.17 \\
Emociones negativas & 3.24 & 0.59 & 3.33 & 0.74 & 3.34 & 0.63 \\
Castigo & 4.89 & 1.12 & 4.49 & 1.47 & 4.66 & 1.47 \\
JR, diálogo & 3.86 & 1.35 & 3.92 & 1.38 & 4.17 & 1.29 \\
JR, responsabilización & 5.14 & 1.16 & 5.29 & 1 & 4.83 & 1.43 \\
JR, reparación & 5.4 & 0.75 & 5.32 & 0.92 & 5 & 1.2 \\
\hline
\end{tabular}

Empatía. Se aplicaron dos indicadores (amabilidad y sufrimiento por empatía) adaptados y validados al castellano por Oceja y Jiménez (2007) a partir del Empathic Response Questionnaire (ERQ, Batson, O'Quin, Fultz, Vanderplas y Isen, 1983). Se utilizó una escala de 7 puntos, donde 1 es "nada en absoluto" y 7 es "totalmente". El índice de confiabilidad para la escala total, constituida por 7 ítems, fue satisfactorio $(\alpha=0.72)$. La amabilidad por empatía se calculó a partir de tres enunciados (por ej. "Por lo general, suelo ponerme en el lugar de las personas incluso aunque no las conozca mucho"). La fiabilidad alcanzó un índice de consistencia elevado (alfa de Cronbach; $\alpha$ $=0.81$ ). A mayor puntuación más sentimientos de amabilidad por empatía $(\mathrm{M}=6.01 ; \mathrm{DE}=$ 0.89). El nivel de amabilidad empática bajo vs. alto se operativizó en función de la mediana (6.25 en la variable amabilidad). Mientras que el sufrimiento por empatía se midió a partir de 4 ítems (por ej. "Ver la desgracia de los otros me pone nervioso"). El alfa de Cronbach fue moderado $(\alpha=0.69)$. A mayor puntuación más sentimientos de sufrimiento por empatía $(M=$ 3.31; DE = 1.27). El sufrimiento empático bajo (vs. alto) se construyó teniendo en cuenta la puntuación de la Mediana $=3$. 
Valores. Para este estudio se acordó la utilización de una versión reducida del Schwartz Value Survey (SVS, Schwartz, 1992), validada por Oceja et al. (2010). Esta escala contiene 6 valores motivacionales que corresponden a los dominios de hedonismo, autodirección, universalismo, tradición, seguridad y quijotismo. Las preguntas se contestan en una escala Likert de 1 (nada importante) a 8 (muy importante), pidiendo al participante que indique la importancia que asigna a cada principio guía en su vida. El alpha de Cronbach que se obtuvo fue adecuado $(\alpha=$ 0.76). A mayor puntuación más importancia a dichos valores $\left[\left(M_{\text {hedonismo }}=7.57 ; D E=.83\right)\right.$, $\left(M_{\text {autodirección }}=6.82 ; \mathrm{DE}=1.31\right),\left(\mathrm{M}_{\text {universalismo }}\right.$ $=6.91 ; \mathrm{DE}=1.41),\left(\mathrm{M}_{\text {tradición }}=6.48 ; \mathrm{DE}\right.$ $=1.53),\left(M_{\text {seguridad }}=6.14 ; D E=1.96\right) \mathrm{y}$ $\left.\left(M_{\text {quijotismo }}=7.53 ; D E=0.99\right)\right]$.

Datos sociodemográficos. Tras la aplicación de todas estas medidas se preguntaba por cuestiones referidas al sexo, edad y situación laboral.

\section{Procedimiento}

A través del programa Qualtrics se recogieron las respuestas de todos los participantes. El cuestionario contenía varias escalas (véase su descripción en el epígrafe instrumentos). Antes de comenzar con la batería de preguntas, se solicitó el consentimiento informado. Por la colaboración voluntaria en el estudio se obtenía una bonificación académica, garantizándose el anonimato y confidencialidad de los datos. Cada estudiante de la Universidad Nacional de Educación a Distancia (UNED) debía contactar con otros sujetos con el fin de obtener una mayor representación de la población española, por lo que la recogida de datos se basó en la técnica de bola de nieve, es decir, se adoptó una estrategia de muestro no probabilístico.

\section{Análisis de Datos}

Se utilizó el paquete estadístico SPSS, versión 24, para realizar los análisis descriptivos [Medias (M) y Desviaciones Estándar (DE)] de las variables objeto de estudio. La comprobación de relaciones propuestas en las hipótesis 1, 2, 4 y 5 se llevó a cabo a través de los análisis correlacionales $r$ de Pearson. Las pruebas univariadas permitieron testar la $\mathrm{H} 3$, donde la baja vs. alta amabilidad empática fue la VI y el diálogo, la responsabilización y la reparación las VD. Para confirmar las comparaciones propuestas en la $\mathrm{H} 5$ se tuvo en cuenta la prueba t-test para muestras relacionadas. Estos análisis se realizaron para cada una de las condiciones (desafiante, arrepentimiento e impunidad). El valor de $p$ para establecer el nivel de significación estadística fue igual o menor de 0.05. Adicionalmente, se efectuaron varios análisis de confiabilidad $\alpha$ Cronbach con el software JASP.

\section{Resultados}

Con el propósito de comprobar que el sexo, la edad y la situación laboral no ejercen ninguna influencia sobre las variables objeto de estudio, se realizaron varios análisis de la varianza. Los resultados nos permiten confirmar que no hubo diferencias significativas respecto al sexo $\left(F_{(1,61)}\right.$ desafiante $=0.23 ; F_{(1,57) \text { arrepentimiento }}=0.22 ; F_{(1,63)}$ impunidad $=0.31 ; F_{(1,185) \text { castigo }}=0.52 ; F_{(1,185)}$ diálogo $=0.59 ; F_{(1,185) \text { responsabilidad }}=0.06$ y $F_{(1,185)}$ reparación $=0.03)$, edad $\left(F_{(32,61)}\right.$ desafiante $=0.95$; $F_{(33,57) \text { arrepentimiento }}=0.79 ; F_{(30,63) \text { impunidad }}=$ $1.23 ; F_{(46,185) \text { castigo }}=0.82 ; F_{(46,185) \text { diálogo }}=0.74$; $F_{(46,185)}$ responsabilidad $=0.88$ y $F_{(46,185)}$ reparación $=$ $0.82)$ y situación laboral $\left(F_{(6,61)}\right.$ desafiante $=1.26$; $F_{(6,57) \text { arrepentimiento }}=1.55 ; F_{(6,63) \text { impunidad }}=1.53$; $F_{(6,185) \text { castigo }}=1.61 ; F_{(6,185) \text { diálogo }}=1.13 ; F_{(6,185)}$ responsabilidad $=1.10$ y $F_{(6,185)}$ reparación $\left.=1.47\right)$. En todos los casos $p>0.05$.

El análisis de correlaciones permitió poner a prueba la primera hipótesis; los resultados constataron asociaciones entre el indicador referido a las emociones negativas que el participante siente tras la resolución del conflicto con las distintas variables que constituyen la JR. Así, a más emociones negativas menor predisposición a hablar con el infractor que ha 
cometido el delito $\left(r_{\text {desafiante }(63)}=-0.41 ; p<\right.$ $0.001 ; r_{\text {impunidad (65) }}=-0.28 ; p<0.05$ ), a la responsabilización $\left(r_{\text {desafiante }}(63)=-0.31 ; p<\right.$ 0.01 y a aceptar la reparación a la víctima por el daño causado como forma de gestionar el delito $\left(r_{\text {desafiante }}(63)=-0.32 ; p<0.01\right)$.

La H2 también fue testada a través del análisis correlacional, los datos confirmaron que los participantes que consideran que el agresor debe cumplir la mayor condena presentan menor predisposición al diálogo $\left(r_{\text {desafiante }}(63)=-0.30\right.$; $p<0.01 ; r_{\text {arrepentimiento (59) }}=-0.61 ; p<0.001 ;$ $\left.r_{\text {impunidad (65) }}=-0.16 ; p<0.05\right)$ у а aceptar las conductas de reparación $\left(r_{\text {desafiante }}(63)=\right.$ -0.25; $p<0.05)$, así como un mayor número de emociones negativas $\left(r_{\text {desafiante }}(63)=0.26 ; p\right.$ $<0.05 ; r$ arrepentimiento (59) $=0.49 ; p<0.001$; $\left.r_{\text {impunidad (65) }}=0.61 ; p<0.001\right)$.

Con el objeto de analizar cómo influye la alta amabilidad empática sobre el proceso de JR se efectuó un análisis univariado. Los resultados constataron, para la condición arrepentimiento, que a más amabilidad empática más predisposición a aceptar la reparación como forma de respuesta ante el delito $\left(M_{\text {alta amabilidad }}\right.$ =5.47, $D E=1.43$ vs. $M_{\text {baja amabilidad }}=4.59$, $\left.D E=1.84 ; F_{(1,57)}=4.21, p<0.05\right)$, a considerar que la asunción de responsabilidad por parte del victimario sea también tenida en cuenta en la gestión del delito $\left(M_{\text {alta amabilidad }}=5.11\right.$, $D E=1.39$ vs. $M_{\text {baja amabilidad }}=4.31, D E=1.45$; $\left.F_{(1,57)}=5.03, p<0.05\right)$ y a imponer una condena menor $\left(M_{\text {alta amabilidad }}=4.00, D E=2.16\right.$ vs. $M_{\text {baja }}$ amabilidad $\left.=5.62, D E=1.9 ; F_{(1,57)}=6.84, p<0.01\right)$. Por lo tanto, la $\mathrm{H} 3$ se confirma en parte, ya que no hubo diferencias estadísticamente significativas para el indicador de JR referido al diálogo entre las partes. Si bien a lo largo del estudio hemos visto cómo las emociones negativas imposibilitan el diálogo, en la $\mathrm{H} 3$ el hecho de que no haya diferencias entre baja vs. alta amabilidad empática no significa que las personas empáticas no estén dispuestas a ponerse en el lugar del otro y mantener una comunicación.

Las relaciones entre el valor social caracterizado por el quijotismo y los 4 indicadores de JR (castigo, diálogo, responsabilización y reparación) fueron estadísticamente no significativos para todas las condiciones objeto de estudio. Por lo que no se confirma la H4.

Para contrastar la H5 se llevó a cabo, en primer lugar, un análisis de comparación intrasujeto para ambos componentes de la empatía a través de la prueba $t$-test. Los resultados confirmaron que en las 3 condiciones del estudio los participantes presentaban más sentimientos positivos de amabilidad que de sufrimiento, siendo todas las diferencias de medias estadísticamente significativas $\left[\left(M=2.88, D E=1.63, t\right.\right.$-test $t_{\text {Desafiante }}$ $(62)=14.03 ; \quad p<0.001), \quad(M=2.78, D E=1.46$, t-test $\left._{\text {Arrepentimiento }} 2{ }_{(58)}=14.56 ; p=<0.001\right) \quad y$ $\left(\mathrm{M}=2.99, \mathrm{DE}=1.55\right.$, t-test $_{\text {Impunidad }}(64)=15.56$; $p<0.001)]$. En segundo lugar, se realizaron análisis de correlaciones: concretamente se vincularon los valores sociales y ambos tipos de empatía (amabilidad y sufrimiento), obteniéndose una asociación significativa y positiva entre los siguientes valores y la amabilidad por empatía $\left(r_{\text {hedonismo (187) }}=0.24\right.$, $p<0.001 ; r_{\text {universalismo (187) }}=0.40, p<0.001$ y $\left.r_{\text {quijotismo (187) }}=0.37, p<0.001\right)$. Mientras que las relaciones negativas se produjeron entre el sentimiento de sufrimiento por empatía y los valores caracterizados por $\left(r_{\text {autodirección (187) }}\right.$

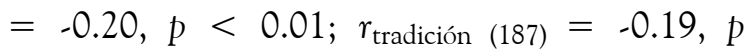
$<0.01$ y $\left.r_{\text {seguridad }(187)}=-0.26, p<0.001\right)$. Estos resultados en su conjunto nos permiten confirmar nuestro planteamiento, de que sólo cierto tipo de empatía se relaciona con unos determinados valores sociales.

Por último, y aunque no teníamos ninguna hipótesis planteada en este sentido, fueron encontradas relaciones positivas entre algunos valores de Schwartz con el indicador que guarda relación con la condena/castigo ( $r_{\text {autodirección (187) }}$ $=0.13, p<0.05 ; r_{\text {tradición (187) }}=0.30, p<$ 0.001 y $\left.r_{\text {seguridad (187) }}=0.20, p<0.01\right)$. Es decir, a mayor puntuación en autodirección, tradición y seguridad, más proclives se encuentran las personas a considerar que el victimario debe cumplir el máximo castigo. Estos hallazgos van en la línea de los resultados anteriores que 
vinculaban dichos valores con el sufrimiento empático.

\section{Discusión}

Esta investigación permite conocer cómo reaccionamos cuando somos conocedores de la vivencia de un delito que ha sufrido otra persona, así como aproximarnos al conjunto de sentimientos negativos que van desde el enfado hasta la incomodidad, siendo el sentimiento de cólera la emoción que más se manifiesta para las tres condiciones del estudio (desafiante, arrepentimiento e impunidad), mientras que la indiferencia junto con la venganza son los afectos que se expresan con una menor intensidad ante el delito que sufre la víctima. Estas emociones negativas afectan la forma en la que creemos que se hace justicia a la hora de gestionar el delito y el conflicto social. Además, generan un rechazo hacia planteamientos restaurativos basados en el diálogo. En esta línea, hay estudios que plantean que el miedo que la víctima siente ante su ofensor, puede llegar a excluir la posibilidad de participar en una actividad restaurativa (Bargen et al., 2018).

Por otra parte, en este ejercicio de percepción de justicia nos vamos a ver influidos por la actitud de quien ha cometido dicho delito. Así, los resultados correlacionales constataron que, cuando se tiene que gestionar un delito cometido por un infractor con una actitud desafiante se van a sentir más emociones negativas, que van a hacer más difícil que se acepten los procesos de JR caracterizados por el diálogo, la responsabilidad y la reparación.

Estudios previos (Barnett, 1977; Christie, 1977; Gustafon, 2018; Varona, 2018; Zernova \& Wright, 2007) indican la existencia de marcadas resistencias por parte de la sociedad respecto a la aceptación de los procesos de JR. Esto se confirma en nuestra investigación cuando los participantes consideran que los agresores deben cumplir el mayor castigo; es más, esta imposición de mayor condena va asociada a un mayor número de emociones negativas, incluso cuando han sido asignados a la condición de arrepentimiento por parte del infractor. Ahora bien, la tendencia a castigar a los infractores como estrategia esencial de prevención, como señalan Sherman y Strang (2007), no permite solucionar el problema de la delincuencia, sino que influye más bien en la percepción de "justicia aplicada", "lo justo" (fairness) y en la falsa ilusión de seguridad ciudadana (Bolívar \& Vanfraechem, 2015; de Mesmaecker, 2011; Umbreit et al., 2005).

La vinculación entre el cumplimiento elevado de la condena y la menor predisposición al diálogo no se produjo para la condición impunidad. Una posible explicación la podemos encontrar en el texto del supuesto ya que, al no haber delincuente, muy difícilmente puede haber diálogo entre las partes sobre un asunto que les ocupa e influye mutuamente.

Respecto a la empatía, investigaciones previas han hallado la relación entre empatía y la tendencia a aceptar soluciones centradas en la víctima (Gault \& Sabini, 2000). Además, y tal y como postula López-Pérez (2012), la amabilidad empática a diferencia del sufrimiento por empatía, posibilita que tengamos conductas más beneficiosas para el conjunto de la sociedad. En el tema que nos ocupa, esto se ve reflejado en la responsabilización de la persona que delinque y en la reparación a la víctima que ha sufrido el delito. Es más, los participantes con alta amabilidad empática están dispuestos a no imponer la mayor condena y a mostrarse abiertos a procesos basados en el diálogo siempre y cuando se dé la responsabilización de la persona ofensora y su propósito de reparar a quien ha dañado. Estos resultados constatan el acercamiento de la cultura de paz a nuestra sociedad y alientan la implementación de procesos de JR.

La reparación a la víctima y su nivel de satisfacción tras participar en conferencias restaurativas se ha constatado en el metaanálisis llevado a cabo por Sherman et al. (2015) que muestra que, en comparación con las víctimas que no han participado en esta práctica de JR, estas expresan niveles más altos de satisfacción en cuanto al manejo de sus casos, son más propensas a recibir y aceptar disculpas por parte del victimario, calificándolas como sinceras y 
a no presentar emociones relacionadas con la venganza.

Así pues, nuestro resultado sobre el sentimiento de venganza va en línea de lo postulado por Bolívar y Vanfraechem, 2015; Braithwaite, 2002; Miguel Barrio, 2019 y Zehr, 2002; al constatar que las personas no necesitamos que la respuesta punitiva al delito implique para el ofensor un perjuicio mayor del que merece.

El último factor psicosocial que hemos abordado en este estudio se refiere a los valores sociales de Schwartz (1992) y al quijotismo de Oceja et al. (2010). La vinculación del quijotismo con los procesos de JR no se ha confirmado. Una posible explicación es que se ha evaluado este indicador como medida disposicional y estable.

Los valores sociales que sí explican, tanto la empatía como los procesos de JR, en el sentido de lo hipotetizado, son la autodirección, la tradición y la seguridad, ya que, a más sufrimiento empático, más tendencia al conservadurismo y a la necesidad de imponer el máximo castigo como respuesta al delito.

Uno de los motivos por los que posiblemente la JR recibe rechazo podría pivotar en el significado otorgado, al entenderse que su aplicación podría suponer impunidad, dejando sin castigo las conductas delictivas. No obstante, la JR es un sistema complementario al sistema de justicia tradicional, y no se trata de un sistema alternativo y excluyente (Ríos et al., 2016).

En conclusión, esta investigación permite sentar un precedente respecto a algunos de los factores psicosociales que pueden explicar la implementación en nuestra sociedad de la JR para gestionar delitos. Igualmente, aporta una valiosa clave a facilitadores y mediadores a la hora de alentar a las personas a su participación en prácticas restaurativas, apelando al bienestar generado por la conexión con otras personas, fruto de la empatía por amabilidad, emoción directamente relacionada con los procesos de diálogo, responsabilización y reparación propios de la JR.

\section{Limitaciones y líneas futuras de investigación}

Una de las primeras limitaciones de este trabajo es el tamaño muestral. Si bien la mayoría de las hipótesis planteadas han recibido apoyo empírico, hay que ser cautos a la hora de realizar una generalización de resultados. Además, los tres supuestos sobre JR planteados y creados exprofeso para este estudio deben ser replicados en futuras investigaciones. Incluso para la condición impunidad habría que matizar la cuestión sobre el posible diálogo entre las partes, tal y como se ha expuesto anteriormente.

Los hallazgos sobre valores sociales generan, por una parte, la necesidad de realizar nuevos estudios donde se manipule el valor del quijotismo y pase a ser una medida situacional, con el fin de comprobar si la persona que tiene como meta mejorar el bienestar del mundo, también está dispuesta a gestionar el delito desde los pilares de la JR. Por otra parte, sientan las bases para el diseño de modelos estructurales que confirmen las relaciones entre la percepción de justicia y seguridad ciudadana con las emociones como moderadores en la implementación de la JR.

Finalmente, una posible línea de investigación sería realizar comparaciones transculturales que nos permitan conocer cómo se relacionan la JR, los valores sociales, la amabilidad y el sufriente empático en diferentes contextos culturales.

\section{Referencias}

Bargen, C., Edwards, A., Hartman, M., Hasslet, J., \& Lyons, A. (2018). Serving Crime Victims through Restorative Justice. A Resource Guide for Leaders and Practitioners. ARJA. https://www.justoutcomesconsultin g.com/wp-content/uploads/SCVTRJG.pdf

Barnett, R. E. (1977). Restitution: A new paradigm of criminal justice. Ethics, 87, 279-301. https://doi.org/10.1086/292043

Batson, C. D. (1991). The altruism question: Toward a social psychological answer. Lawrence Erlbaum. 
Batson, C. D., O'Quin, K., Fultz, J., Vanderplas, M., \& Isen, A. (1983). Self-reported distress and empathy and egoistic versus altruistic motivation for helping. Journal of Personality and Social Psychology, 45, 706-718. https:// doi.org/10.1037/0022-3514.45.3.706

Bolívar, D., \& Vanfraechem, I. (2015). Víctimas en justicia restaurativa: isujetos activos o en necesidad? Un estudio europeo desde la perspectiva de operadores sociales. Universitas Psychologica, 14(4), 1437-1458. https://doi.org/10.11144/Javeri ana.up14-4.vjrs

Braithwaite, J. (1999). Restorative justice: Assessing optimistic and pessimistic accounts. En M. Tonry (Ed.), Crime and justice: A review of research (Vol. 25, pp. 1127). University of Chicago Press.

Braithwaite, J. (2002). Setting standards for restorative justice. The British Journal of Criminology, 42, 563-577. https://doi.org/1 0.1093/bjc/42.3.563.

Chapman, T., Van Hoek, A., \& Wolthuis, A. (2018). The road less travelled. More human approaches to addressing the harm of criminal behavior. Restorative Justice Nederland. https://www.euforumrj.org/site s/default/files/2020-01/the-road-less-travell ed-2018.pdf

Christie, N. (1977). Conflicts as property. The British Journal of Criminology, 17, 1-15. https://doi.org/10.1093/oxfordjournal s.bjc.a046783

de Mesmaecker, V. (2011). Perceptions of justice and fairness in criminal proceedings and restorative encounters: extending theories of procedural justice. [Doctoral dissertation, KU Leuven Faculty of Law]. https://core.ac .uk/download/pdf/34510494.pdf

Echebarría, A., \& Páez, D. (1989). Emociones, perspectivas psicosociales. Fundamentos.

Fernández, I. (2012). El estudio de la Cultura desde la Psicología Social. En I. Fernández \& I. Cuadrado (Coords.), Psicología Social: 2a Edición (pp. 33-70). UNED/Sanz \& Torres.

Garrido Genovés, V. (2010). La prevención de la delincuencia en Europa y en España: los retos pendientes. Revista de Derecho Penal y Criminología, 3, 377-408. http://revistas.un ed.es/index.php/RDPC/article/view/24638

Gault, B. A., \& Sabini, J, (2000). The roles of empathy, anger, and gender in predicting attitudes towards punitive, reparative and preventative public policies. Cognition and Emotion, 14, 495-520. https://doi.org/10.10 80/026999300402772

Gustafson, D. L. (2018) Encountering "the Other": Victim Offender Dialogue in Serious Crime. [Doctoral dissertation, KU Leuven Faculty of Law]. https://lirias.kuleuven.be/1996032 ?limo $=0$

Hofinger, V., Lauwaert, K., \& Marsh, B. (2015) Guidance for developing restorative justice processes supporting desistance. Promising practices. European Forum for Restorative Justice. Leuven. http://www.euforumrj.org/ wp-content/uploads/2015/11/Guidance-for -developing-RJ-supporting-desistance-boo klet-format-12-11-final.pdf

Johnstone, G. (2013). Restorative justice: Ideas, values, debates. Routledge.

Lishner, D. A, Batson, C.D., \& Huss, E. (2011). Tenderness and sympathy: distinct empathic emotions elicited by different forms of need. Personality and Social Psychology Bulletin, 37, 614-625. https://doi .org/10.1177/0146167211403157

López-Pérez, B. (2012). Caracterización de la experiencia emocional vicaria inducida por la percepción de necesidad: compasión, ternura y estrés. [Tesis Doctoral]. Universidad Autónoma de Madrid.

López-Pérez, B., Carrera, P., Oceja, L., Ambrona, T., \& Stocks, E. (2019). Sympathy and Tenderness as components of dispositional empathic concern: Predicting helping and caring behaviours. Current Psychology 38, 458-468. https://doi.org/10.1007/s12144-0 $17-9615-7$

Maglione, G. (2017). Embodied victims: An archaeology of the "ideal victim" of restorative justice. Criminology and Criminal Justice, 17, 401-417. https://doi.org/10.1177 $/ 1748895816677172$ 
Marshall, T. F. (1999). Restorative justice: An overview. Home Office.

Miguel Barrio, R. (2019). Justicia Restaurativa y Justicia Penal. Atelier.

Oceja, L., Ambrona, T., López-Pérez, B., Salgado, S., \& Villlegas, M. (2010). When the victim is one among others: Empathy, awareness of others and motivational ambivalence, Motivation and Emotion, 34, 110-119. https: //doi.org/10.1007/s11031-010-9161-1

Oceja, L., \& Jiménez, I. (2007). Beyond egoism and group identity: Empathy toward the other and awareness of others in a social dilemma. The Spanish Journal of Psychology, 10, 369-379. https://doi.org/10.1017/S1138 741600006636

Olalde, A. (2015). Estudio multidimensional de algunas prácticas de Justicia Restaurativa en el País Vasco con Lentes de Trabajo Social (2007-2012). [Tesis doctoral]. Universidad de Murcia.

Páez, D., Fernández, I., Ubillos, S., \& Zubieta, E. (2003). Psicología Social, Cultura y Educación. Madrid: Pearson/Prentice Hall.

Páez, D., Vázquez, C., Bosco, S., Gasparre, A., Iraurgi, I., \& Sezibera, V. (2011). Crecimiento post-estrés y post-trauma: posibles aspectos positivos y beneficiosos de la respuesta a los hechos traumáticos En D. Páez, C. Martin Beristaín, J.L. González, N. Basabe, \& J. de Rivera, (Eds.). Superando la Violencia Colectiva y Construyendo Cultura de Paz (pp. 311-342). Madrid: Fundamentos.

Pascual, E. (2012). La mediación en el sistema penal. Propuestas para un modelo reparador, humano y garantista. [Tesis doctoral]. Madrid: Universidad Complutense. https:// eprints.ucm.es/16592/1/T33979.pdf

Pascual, E. (2013). Los ojos del otro. Encuentros restaurativos entre víctimas y ex miembros de ETA. Madrid: Sal Terrae.

Ríos, J. C., Pascual, E., Segovia, J. L., Etxebarria, X., \& Lozano, F. (2016) Mediación penal, penitenciaria y encuentros restaurativos. Experiencias para reducir el sufrimiento en el sistema penal. Madrid: Universidad Pontificia de Comillas.
Salgado, S. (2009). Caracterización teórica y comprobación experimental de un nuevo motivo social, el quijotismo, [Tesis Doctoral]. Madrid: UAM.

Schwartz, S. H. (1992). Universals in the content and structure of values: Theoretical advances and empirical tests in 20 countries. Advances in Experimental Social Psychology, 25, 1-65. https://doi.org/10.101 6/S0065-2601(08)60281-6

Schwartz, S. H., \& Bilsky, W. (1990). Towards a theory of the universal content and structure of values: Extensions and crosscultural replications. Journal of Personality and Social Psychology, 58, 878-891. https://d oi.org/10.1037/0022-3514.58.5.878

Sherman, L.W., \& Strang, H. (2007). Restorative Justice. The evidence. Londres: The Smith Institute.

Sherman, L.W., Strang, H., Mayo-Wilson, E., Woods, D. J., \& Ariel, B. (2015). Are Restorative Justice Conferences Effective in Reducing Repeat Offending? Findings from a Campbell Systematic Review. Journal of Quantitative Criminology, 31, 1-24, https://d oi.org/10.1007/s10940-014-9222-9

Staiger, I. (2008). Restorative justice and victims of terrorism. About the Project. European Forum for Restorative Justice. Newsletter 9, 1-2. https://www.euforumrj.org/

Umbreit, M. S., Vos, B., Coates, R. B., \& Lightfoot, E. (2005). Restorative justice in the twenty-first century: A social movement full of opportunities and pitfalls. Marquette Law Review, 89, 251-304. https://scholarship.law.marquette .edu/mulr/vol $89 /$ iss $2 / 3$

United Nations. (2006). Handbook on restaurative justice programmes. Nueva York: United Nations Office on Drugs and Crime. https://www.unodc.org/documents/ justice-and-prison-reform/Manual_sobre_p rogramas_de_justicia_restaurativa.pdf

Varona, G. (2018). Justicia restaurativa desde la Criminología: Mapas para un viaje inicial. Madrid: Dykinson.

Zehr, H. (2002). The little book of restorative justice. Pennsylvania: Good Books. 
Zehr, H. (2005). Evaluation and restorative justice principles. En E. Elliot \& R. M. Gordon (Eds.), New Directions in Restorative Justice: Issues, Practice, Evaluation (pp. 296-303). Cullompton: William Publishing. https://doi.org/10.4324/9781843926429

Zernova, M., \& Wright, M. (2007). Alternative visions of restorative justice. En G. Johnstone, \& D. W. Van Ness (Eds.), Handbook of restorative justice (pp. 91-108). Cullompton: William Publishing.

\section{Apéndice A}

\section{Descripción de las condiciones desafiante, arrepentimiento e impunidad}

Un amigo tuyo te cuenta que ha sufrido un delito. Te relata que un día por la calle iba chateando con su teléfono móvil, ajeno a lo que ocurría a su alrededor. De repente, sufre un empujón que le hace caer al suelo y alguien que intenta arrancarle el móvil de sus manos. Se trataba de un iphone que se acababa de comprar y que trató de no soltar, pero estaba adolorido por la caída y al final esa persona consigue hacerse con él y salir corriendo. Tu amigo te aclara que fue a comisaría a poner la denuncia y al hospital, donde le escayolaron el brazo izquierdo debido al golpe que recibió cuando cayó al suelo. Posteriormente, se fue recuperando según pasaron las semanas, sin saber nada acerca del suceso ni de la persona hasta que...

Condición desafiante: le avisan que se inicia el proceso judicial porque han encontrado a la persona que le robó el móvil. Te pide si le puedes acompañar al juicio, puesto que está preocupado por cómo será la experiencia, ya que nunca antes había participado en un juicio.

Cuando estás allí, te encuentras a una persona con actitud desafiante, que te mira a ti y a tu amigo con actitud amenazante, sin ningún rastro de arrepentimiento.

Esta condición hace referencia a la percepción externa de una actitud retadora, por la cual da a entender quien la exhibe, una falta de responsabilización por el daño causado y de empatía hacia quien lo ha sufrido, así como la ausencia de intención reparadora.

Condición arrepentimiento: le avisan que se inicia el proceso judicial porque han encontrado a la persona que le robó el móvil. Te pide si le puedes acompañar al juicio, puesto que está preocupado por cómo será la experiencia, ya que nunca antes había participado en un juicio.

Cuando estás allí, te encuentras a una persona con actitud de arrepentimiento. Su abogado de oficio expresa que su cliente desearía pedir perdón a tu amigo, e intentar repararle.

Esta condición alude a la actitud de pesar por parte de quien ha cometido el daño, consciente de las consecuencias negativas generadas en otros, debido a sus actos. En este caso, la persona manifiesta además su propósito de enmendar el daño a través de la disculpa y reparación.

Condición impunidad: entendió que el delito había quedado impune, ya que nunca tuvo noticias acerca del tema.

La condición impunidad en este estudio se refiere a la situación por la que un delito no obtiene una respuesta por parte de la justicia, debido a diferentes causas posibles; esta situación arroja como resultado la falta de medidas hacia quien comete el delito, así como la desatención de las necesidades generadas en la persona que ha sufrido las consecuencias del mismo.

\section{Notas}

* Artículo de investigación. 